\title{
Alpha-linolenic acid content and expression of KASII and FAD3 in perilla seed associated with altitude of cultivation areas
}

\author{
Orada Chumphukam ${ }^{\mathrm{a}}$, Wachiraporn Tipsuwan ${ }^{\mathrm{a}}$, Chakkrit Khanaree ${ }^{\mathrm{a}, \mathrm{b}}$, Komsak Pintha ${ }^{\mathrm{a}}$, \\ Payungsak Tantipaiboonwong $^{\mathrm{a}}$, Wittaya Chaiwangyen ${ }^{\mathrm{a}}$, Sitiruk Roytrakul ${ }^{\mathrm{c}}$, Maitree Suttajit ${ }^{\mathrm{a}}$, \\ Supachai Topanurak ${ }^{\mathrm{d}, *}$ \\ a Division of Biochemistry, School of Medical Sciences, University of Phayao, Phayao 56000 Thailand \\ b Department of Biochemistry, Faculty of Medicine, Chiang Mai University, Chiang Mai 50200 Thailand \\ c Genome Institute, National Center for Genetic Engineering and Biotechnology, Science Park, \\ Pathum Thani 12120 Thailand \\ d Department of Molecular Tropical Medicine, Faculty of Tropical Medicine, Mahidol University, \\ Bangkok 10400 Thailand
}

*Corresponding author, e-mail: Supachai.top@mahidol.ac.th

Received 18 Sep 2018

Accepted 23 Sep 2019

\begin{abstract}
Perilla frutescens is commonly used as an n-3-fatty acid source for people living in Northern Thailand. However, cultivated areas are limited because it apparently requires specific growth conditions. Our results suggested that perilla seeds grown at higher altitude in Maehongson province contained more $\alpha$-linoleic acid (ALA) contents than seeds collected from Chiang Rai and Nan provinces. Furthermore, mass spectrometry, proteomic and gene expression analysis revealed that the increase of KASII and FAD3 proteins was associated with mRNA expression and ALA production. Predictive bioinformatics analysis demonstrated two important transcription factor binding sites, AP2 and B3, are responsible for kasII and fad3 genes, respectively. The AP2 and B3 transcription factor families were known to be responsible for abiotic stress such as drought and temperature changes. From our observation, geographical factors could possibly influence the expression of genes related to fatty acid production. Furthermore, plant responses to abiotic stress environments contribute to the increase of kasII and fad3 genes and their protein expression. However, responsive transcription factors will be further studied as well as other geographical influences for a proof of concept and also to improve growing methods in order to increase perilla productivity.
\end{abstract}

KEYWORDS: Perilla frutescens, n-3 fatty acids, proteomics, kasII, fad3

\section{INTRODUCTION}

Perilla frutescens, also called perilla, is a member of the mint families, Lamiaceae. Perilla is commonly known as a rich source of unsaturated fatty acids, especially $\alpha$-linolenic acid (ALA), which is comparable to other oilseeds such as flax (Linum usitatissimum) and inca peanut (Plukenetia volubilis) ${ }^{1}$. The ALA is a precursor for other n-3 fatty acids biosynthesis in the human body. Once obtained from food sources, it can be further metabolized into eicosapentaenoic acid (EPA) and docosahexaenoic acid (DHA) by the action of $\Delta 12$ and $\Delta 15$ desaturases ${ }^{2}$. Perilla oil contains as much as $50-65 \%$ ALA $^{1,3}$ and thus it can be promoted as a part of diets. A regular consumption of food containing high n-3 fatty acids is beneficial in terms of promoting health and preventing illness. For example it may reduce the risk of cardiovascular disease ${ }^{4}$ and other noncommunicable diseases ${ }^{5}$ as well as help the brain function in learning and memory ${ }^{6,7}$.

From a practical point of view, the n-3 fatty acids from plants are not only a benefit for direct consumption but can also potentially be promoted as food supplements. Increased consumption of n3 fatty acids can drive the n6:n3 imbalance ratio in the modern diet to a more proper proportion where the ideally recommended ratio is $1: 1^{5}$. The $n-3$ fatty acid producing plants are also recognized as an alternative to fish oil, and thus they are becoming a valuable choice for vegetarian diets ${ }^{3}$. Furthermore, the importance of n-3 fatty acids is not only in foodbased ingredients, but it is also widely used as a component, primarily from virgin flaxseed oils, in cosmetics and personal care products as part of their 
lipid-based formulations. On the other hand, perilla seed oil has not been reported to be used on an industrial scale. This could be due to its limited availability. Important driving factors that have limited its widespread use may include restrictively cultivated lands, distinctive growing conditions as well as the efficiency of a plantation that can produce effective crop yield.

In Thailand, perilla cultivated areas are relatively limited. A perilla commonly grows well in mountain areas because the average annual temperature is relatively low, and the humidity is appropriate. Apparently, the ability of perilla to produce ALA varies in each cultivation area. Our previous study on fatty acids and the nutrients of perilla seeds revealed that the ALA content of perilla seed grown in Maehongson was the highest among mature dry seeds from 14 cultivation areas ${ }^{8}$. In this study, we determined whether ALA contents in mature perilla dry seeds from 3 locations, Maehongson, Chiang Rai and Nan, are different in relation to the altitudes of each cultivation area, particularly in the aspect of gene expression. In general, many genes encoding enzymes in lipid biosynthesis are temporally up-regulated during oilseed development ${ }^{9,10}$. The key genes involved in n-3 fatty acid biosynthesis, $\beta$-ketoacyl-acyl carrier protein synthase II (kasII) and fatty acid desaturase-3 (fad3), were selected as candidates for surrogate markers in this study. The kasII expression is not a tissue-specific. Its basal expression level can be found throughout all developmental stages of both perilla leaves and seeds ${ }^{11}$. While the expression of fad 3 has a seedspecific expression ${ }^{12}$. There were also reports showing that the expression of fad3 was higher in developing seeds than in leaves ${ }^{9,13}$. Indeed, at a certain time, gene expression would not be a sole indicator representing ALA production. Proteins are effective molecules affecting enzymatic reactions in perilla seeds. Hence not only mRNA transcripts but also protein levels were determined in order to answer question of whether protein expression correlated with mRNA abundance is associated with ALA content. Quantitative PCR and quantitative mass-spectrometry-based proteomics were applied to determine both mRNAs and proteins levels. Furthermore, this finding can be useful for crop modification in order to increase crop yield. Nevertheless, these surrogate markers still need to be validated in further study.

\section{MATERIALS AND METHODS}

\section{Chemicals and sample collection}

All chemicals used were reagent grade except that the chemicals used in RNA and DNA work were molecular grade and trypsin was sequencing grade. Mature perilla seeds (grown and collected in field conditions) were collected from Maehongson, Chiang Rai, and Nan provinces. Seeds were carried on dry ice and kept at $-80^{\circ} \mathrm{C}$ for further RNA extraction, while seed samples for lipid analysis were sundried prior to oil extraction. Inca peanut was a gift from Chiang Rai Agriculture Development Co., Ltd. Flax seeds were obtained from the Royal Project Doi Kam, Chiang Rai.

\section{Fatty acid composition and lipid analysis}

Two kilograms of sun-dried seed samples from each location were subjected to oil extraction using the cold-pressed method. The raw perilla seeds were pressed directly through a stainless steel expeller at room temperature and the temperature of the machine during the process did not exceed $50^{\circ} \mathrm{C}$. Oil was then centrifuged at $4000 \mathrm{~g}$ for $10 \mathrm{~min}$ to remove all other unwanted residues. Yellowish pure perilla oil was kept at $4^{\circ} \mathrm{C}$ for further investigation. Fatty acid compositions were analysed using GC/MS (Agilent Technologies) by the Institute of Product Quality and Standardization, Maejo University.

\section{RNA extraction and cDNA preparation}

The total RNA was extracted from seed samples as described previously ${ }^{14}$. In brief, $30 \mathrm{mg}$ of seeds were mixed in an equal amount with PVP40. Seeds were then ground in liquid nitrogen before adding extraction buffer ( $8 \mathrm{M} \mathrm{LiCl}, 2 \%$ $(\mathrm{w} / \mathrm{v})$ PVP-40 and 5\% (v/v) mercaptoethanol) and ethanol. Chloroform was used in order to remove high molecular weight impurities and lipids. The pellet was re-dissolved in solubilization buffer (1\% (w/v) SDS, $0.75 \mathrm{M} \mathrm{NaCl}, 0.025 \mathrm{M}$ EDTA, 2\% (v/v) mercaptoethanol) and chloroform extraction was repeated followed by centrifugation at $4600 \mathrm{~g}$ for $3 \mathrm{~min}$. The supernatant was transferred to a new microcentrifuge tube and gently mixed with TRI reagent (Molecular Research Center). Centrifugation at $26600 \mathrm{~g}$ for $2 \mathrm{~min}$ was carried out and the aqueous phase was transferred to a new tube for RNA precipitation using isopropanol. RNA pellets were then resuspended in RNase-free water. The cDNA was prepared using ReverTra Ace qPCR RT kit (TOYOBO) and kept at $-20^{\circ} \mathrm{C}$ until use. 


\section{Gene selection and primer design}

Two genes associated with fatty acid biosynthesis were selected in this study. Conserved regions of selected genes were compared with other plant species on the NCBI database using CLUSTAL omega multiple sequence alignment. The kasII was compared with plant species including Perilla frutescens, Sesamum indicum, Jatropha curcas, Theobroma cacao, and Glycine max. The fad3 was compared with plant species including $P$. frutescens (Accession numbers: U59477, AF213482, AF047039, and KC990786), $S$. indicum, Nicotiana tabacum, Camelina sativa, and Solanum tuberosum. Elongation factor- $1 \alpha$ gene (ef$1 \alpha$ ) was used as a constitutive control gene. Primer pairs for sequence analysis were designed using Primer3 software ${ }^{15}$. The sequences of forward $(\mathrm{F})$ and reverse $(\mathrm{R})$ primers were as follows: ef- $1 \alpha \mathrm{F}: 5^{\prime}$ TACTACTGCACTGTBATTGATGC- $3^{\prime}$ R: $5^{\prime}$-CAATCT TGTANACRTCCTGAAGTG-3', kasII F: 5'-ATACCHA TTGGDTTGGGAGG-3' R: $5^{\prime}$-CRATCATDGAYTTTG TRGARTTCAC-3' , fad3 F: 5' -CTTCAACCCTTACAGC GATTTG-3' R: 5' The underline bases were degenerative bases which were B: G/C/T, D: A/G/T, H: A/C/T, N: A/G/C/T, R: $\mathrm{A} / \mathrm{G}$ and $\mathrm{Y}: \mathrm{C} / \mathrm{T}$. Gene-specific primers for real-time PCR were designed for the gene length between 100 and $150 \mathrm{bp}$. The sequences of forward and reverse primers were as follows: ef- $1 \alpha \mathrm{F}$ : $5^{\prime}$-CAAGGATGGT CAGACACGTGA-3' R: 5' ${ }^{\prime}$-TCATCGTACCTTGCCTTTG AGT-3', kasII F: 5' -ATGGGAGAAGGTGCTGGAGT-3' R: 5'-CTTGTGGATGAGGCTCTGTCA-3', fad3 F: 5'-C TTCAACCCTTACAGCGATTTG-3' R: 5' -GGTACGCC GTAGAGCTTGA-3'.

\section{Gene cloning and sequence analysis}

Amplification was carried out at the optimum condition for each gene using the following temperature conditions: 1 cycle of pre-incubation at $95^{\circ} \mathrm{C}$ for $3 \mathrm{~min}$, followed by 30 cycles of denaturation at $95^{\circ} \mathrm{C}$ for $30 \mathrm{~s}$, annealing at $60^{\circ} \mathrm{C}$ for $30 \mathrm{~s}$, and extension at $72{ }^{\circ} \mathrm{C}$ for $30 \mathrm{~s}$. The degenerative primers were used at $1 \mu \mathrm{M}$. The PCR products were analysed on 2\% agarose gel electrophoresis in $1 \times \mathrm{TBE}$ buffer and visualized by RedSafe gel staining (iNtRON Biotechnology). The purified PCR products were cloned into pTZ57R/T TA cloning vector (InsTAclone, Thermo Fisher Scientific). Five selected clones of each gene were sequenced using the service from Macrogen Inc. DNA sequence data were analysed using CLUSTAL omega multiple sequence alignment.

\section{Gene expression analysis}

To evaluate the expression of genes related to fatty acid biosynthesis, a real-time PCR was conducted with THUNDERBIRD SYBR qPCR mix (TOYOBO). Each reaction consisted of $1 \times$ SensiFAST SYBR LoROX mix, $0.3 \mu \mathrm{M}$ of each primer, $1 \times$ ROX dye, and $5 \mu \mathrm{l}$ of a 10-time dilution of cDNA sample in a total volume of $20 \mu \mathrm{l}$. The reactions were run in triplicate on the Applied Biosystems 7500 real-time PCR system (Thermo Fisher Scientific). The PCR program was set at the following conditions: 1 cycle of polymerase activation at $95^{\circ} \mathrm{C}$ for $2 \mathrm{~min}$ followed by 40 cycles of denaturation for $5 \mathrm{~s}$ at $95^{\circ} \mathrm{C}$, then annealing and extension at $60^{\circ} \mathrm{C}$ for $35 \mathrm{~s}$. The data were exported as threshold values (Ct) and further analysed as fold change. The expression of ef-1 $\alpha$ was used as a reference gene.

\section{Proteomic approach for protein expression analysis}

Proteins were extracted by grinding perilla seeds in liquid nitrogen. Then, 1\% SDS was added to the debris to dissolve insoluble proteins, followed by shaking at $37^{\circ} \mathrm{C}$ overnight. Next, the protein solution was centrifuged at $26600 \mathrm{~g}$ for $15 \mathrm{~min}$ and the supernatant was collected. After that $10 \%$ trichloroacetic acid in acetone was added until the final concentration reached $1 \%$. The mixture was then kept at $-20^{\circ} \mathrm{C}$ for at least overnight. The protein pellet was collected by centrifugation at $26600 \mathrm{~g}$ and $4^{\circ} \mathrm{C}$ for $15 \mathrm{~min}$, then dissolved in cold acetone, vortexed vigorously to wash the remaining trichloroacetic acid and kept at $-20^{\circ} \mathrm{C}$ for $30 \mathrm{~min}$. The protein pellet was again collected by centrifugation at $26600 \mathrm{~g}$ at $4{ }^{\circ} \mathrm{C}$ for $15 \mathrm{~min}$ and air-dried for at least $5 \mathrm{~min}$ before dissolving in the solution containing $8 \mathrm{M}$ urea, $10 \mathrm{mM} \mathrm{AmBiC}$, and 1\% SDS. The protein concentration was determined using the standard BCA assay.

\section{Protein electrophoresis and in-gel digestion}

Thirty micrograms of perilla protein extract was run in SDS-PAGE at $30 \mathrm{~mA}$ for $120 \mathrm{~min}$. The separating gel was sliced into 12 small pieces along protein lanes. SDS and Coomassie dye in protein slices were washed by soaking gel slices in 50\% acetonitrile in $25 \mathrm{mM}$ AmBic buffer for $2 \mathrm{~h}$. The solution was removed, and $100 \%$ acetonitrile was added several times until the gel pieces were clear. After that gel slices were incubated in $100 \mu \mathrm{l}$ of $4 \mathrm{mM}$ dithiothreitol in $50 \mathrm{mM}$ AmBic at $60^{\circ} \mathrm{C}$ for $15 \mathrm{~min}$. Then $20 \mathrm{mM}$ iodoacetamide was added 
into the gel slices and further incubated at room temperature for $1 \mathrm{~h}$. The gel washing process was then repeated by adding $50 \%$ acetonitrile in $25 \mathrm{mM}$ AmBic buffer followed by $100 \%$ acetonitrile. After the gel slices became opaque, they were air-dried. These processes were repeated three times. Twenty micrograms of trypsin (Sigma) were added into the gel slices and further incubated overnight. To extract tryptic-digested peptides, $100 \mu 1$ of $75 \%$ acetonitrile in $1 \%$ trifluoroacetic acid was added to the gel slices and incubated for $5 \mathrm{~min}$ in a shaker. The solution was then transferred into a new clean tube. The extracted peptides were dried in SpeedVac. The sample was kept at $-80^{\circ} \mathrm{C}$ for further analysis.

\section{Mass spectrometry analysis}

Peptide samples were dissolved in $0.1 \%$ formic acid. The solutions were analysed using the HCTultra PTM Discovery System (Bruker Daltonics) equipped with the UltiMate 3000 LC System (Dionex). The digested peptides were separated by a nanocolumn (PepSwift monolithic column $100 \mu \mathrm{m}$ i.d. $\times 50 \mathrm{~mm}$ ) in which the mobile phase system was comprised of $0.1 \%$ formic acid (Eluent A) and $80 \%$ acetonitrile in $0.1 \%$ formic acid (Eluent B). An elution was performed for 13 min using a linear gradient from $10 \%$ to $70 \%$, Eluent B at a flow rate of $300 \mathrm{nl} / \mathrm{min}$, followed by a regeneration step (90\% Eluent B) and an equilibration step (10\% Eluent B). Peptide fragment mass spectra were acquired in datadependent AutoMs (2) mode with a scan range of $300-1500 \mathrm{~m} / \mathrm{z}, 3$ averages, and up to 5 precursor ions selected from the MS scan $50-3000 \mathrm{~m} / \mathrm{z}$.

\section{Protein identification, database search and protein abundance quantification}

The LC-MS raw data sets from gel pieces were converted to a Mascot generic file (mgf file) and subjected to a search with an in-house MASCOT search engine (Matrixscience, licensed for Faculty of Tropical Medicine, Mahidol University) against the Arabidopsis thaliana Swissprot database. Search parameters were set as follows, enzyme: trypsin, fix modification: carbamidomethyl at cysteine residue (57.03 Da), variable modification: oxidation at methionine (32 Da), peptide tolerance: $20 \mathrm{ppm}$, MS/MS tolerance: $0.25 \mathrm{Da}$, peptide charge was set at $1+, 2+, 3+$, and monoisotopic mode. The identified protein pass through score 100 lists containing y, b-ion peak lists, and intensity of precursor mass were exported into DAT files and subjected to SKYLINE 4.0 for use as library spectra, then quantification of targeted proteins was performed with the
Table 1 Perilla cultivation areas.

\begin{tabular}{lcrrc}
\hline $\begin{array}{l}\text { Field } \\
\text { location }\end{array}$ & $\begin{array}{c}\text { Latitude/ } \\
\text { Longtitude }\end{array}$ & $\begin{array}{c}\text { Altitude } \\
(\mathrm{m})\end{array}$ & $\begin{array}{c}\text { Temp. } \\
\left({ }^{\circ} \mathrm{C}\right)\end{array}$ & $\begin{array}{c}\text { ALA cont. } \\
\text { (wt.\%) }\end{array}$ \\
\hline Maehongson & $19^{\circ} 25^{\prime} \mathrm{N} / 97^{\circ} 59^{\prime} \mathrm{E}$ & 1164 & 26.9 & $79.3 \pm 4.4$ \\
Chiang Rai & $20^{\circ} 8^{\prime} \mathrm{N} / 99^{\circ} 49^{\prime} \mathrm{E}$ & 431 & 25.5 & $69.1 \pm 5.5$ \\
Nan & $18^{\circ} 34^{\prime} \mathrm{N} / 100^{\circ} 52^{\prime} \mathrm{E}$ & 207 & 27.3 & $63.3 \pm 7.3$ \\
\hline
\end{tabular}

MS1 filtering method.

\section{Protein similarity search and transcription factor binding site search}

Perilla KASII (O48943) and FAD3 (Q92PP7) protein sequences were retrieved from Uniprot (Swissprot) database and multiple aligned by T-coffee algorithm (multiple sequence alignment program). To predict transcription factor binding region, upstream sequences of model A. thaliana of kasII (AT1G74960 $1.0 \mathrm{~kb}$ ) and fad3 (AT2G29980 1.0kb) were retrieved from ThaleMine database ${ }^{16}$ and analysed for transcription factor binding site prediction against Plant Transcription Database with a transcription factor binding site algorithm ${ }^{17,18}$. The threshold $p$-value was set up at less than $1 \times 10^{-6}$. The pair wise matrix score was also derived from the PlantRegMap database.

\section{Statistics}

Gene expression data were expressed as mean \pm SE of duplicate experiments. Statistical significance was calculated using one-way ANOVA.

\section{RESULTS}

\section{Fatty acid compositions in perilla oil}

Perilla is generally grown during May-July of each year and mature seeds were collected in December. Our previous study (unpublished data) revealed that perilla seed samples from Maehongson and Nan are the acuta variety and the sample from Chiang Rai is the arguta variety. Perilla seed variety acuta is collected 7 months after planting, while seed variety arguta is collected 8 months after planting. The lipid content ranged from $28-37 \%$ (g per $100 \mathrm{~g}$ seeds). The ALA content of perilla seeds collected from Maehongson, Chiang Rai, and Nan were $79.3 \pm 4.4 \%$, $69.1 \pm 5.5 \%$, and $63.3 \pm 7.3 \%$, respectively. The $n-6$ linoleic acid (LA) content was found to be $10-20 \%$, and it was presented in a reverse order to the ALA content where the higher the ALA level, the lower the linoleic acid content available. The physical characteristics of seeds, oil content, and growing conditions in the local field (such as geographical height and average annual growth temperature) 
information is derived from the National Statistical Office of Thailand year 2016 and is summarized in Table 1.

\section{mRNA expression of kasII and fad3 and the ALA production}

Mature perilla seeds from three locations were selected for mRNA extraction. The expression of genes encoding kasII and fad 3 was analysed using realtime PCR. The result indicated that seed samples from Maehongson showed significantly higher expression of kasII by $3.4 \pm 0.3$ fold $(p<0.01)$ relative to the expression of genes from the Nan sample (control); while the sample from Chiang Rai showed similar expression to the control (Fig. 1a). However, the expression of fad 3 showed no significant differences among all areas (Fig. 1b). Based on ALA rich plants ${ }^{1,10}$, inca peanut and flax were selected for further analysis to compare the expression of kasII and $\mathrm{fad} 3$ related to ALA production with perilla seed from Maehongson. As shown in Fig. 2, there were no significant differences in the level of expression of genes encoding kasII and fad 3 among three seed oils.

\section{Protein expression of KASII and FAD3 by MS-based proteomics}

The expression of gene encoding fad3 may reflect the activity of n-3 desaturase as well as an effect on the accumulation of ALA contents in various oil crops. The mRNA expression levels do not always correlate to protein expression levels because of transcriptional regulation, post-translational regulation, and mRNA stability. Hence we further examined whether protein expression levels correlate to mRNA expression. Herein, KASII and FAD3 protein abundance in perilla seeds were analysed with LC MS/MS and subsequently quantified by the MS1 filtering method using Skyline software.

MS1 filtering relied on quantification of the identified peptides from LC MS/MS. The tryptic peptides peak areas of KASII from the Maehongson perilla seed samples, FMLYMLTAGA and GFVMGEGAVLLEELEHAK, were higher than in the Chiang Rai and Nan samples (Fig. 3). The peak areas of FAD3 tryptic peptide from Maehongson perilla seeds (Fig. 4), SGADGEVFDGQQQYEGIGK, were also higher than in Chiang Rai and Nan samples. Presumably, the geography of perilla cultivation influenced the expression of genes involving n-3 fatty acid production pathways. Altitudes have been reported to be associated with abiotic stress and related to factors including drought, humidity variation, and low temperatures. Hence the expression of kasII and fad 3 are postulated to be associated with abiotic stress regulatory-transcription factors. The prediction for putative abiotic stress transcription factor binding sites of the promoter of kasII and fad3 upstream region was then carried out to support our hypothesis.

\section{Bioinformatics analysis for transcription factor binding sites}

For the prediction of the regulatory-transcription factors, which are responsible for the correlation of altitudes and their targeted gene expressions, a transcription factor target within $1.0 \mathrm{~kb}$ upstream sequence regions of the targeted genes was investigated. However, $P$. frutescens genome sequencing is incomplete, so far. Hence the A. thaliana completed genome sequences were used as a plant model to identify which transcription factor binding sites are located on the upstream region of kasII and fad3. We then examined whether kasII and fad3 are conserved genes among oil crops and a completed genome sequencing plant model. Firstly, we performed multiple alignment of two proteins among $P$. frutescens, A. thaliana and other oil crops.

Multiple alignment of KASII protein sequences from various oil crops showed nearly 95\% similarity (Fig. 5). Furthermore, multiple sequence alignment of FAD3 protein sequences demonstrated approximately 95-98\% similarity (Fig. 6); particularly, both KASII and FAD3 demonstrated almost $100 \%$ similarity to $A$. thaliana. Hence it was postulated that upstream regions of genes are highly conserved. The upstream $1.0 \mathrm{~kb}$ sequence regions of KASII and FAD3 were derived and predicted with the prediction tool in Plant Transcription Database. The prediction criteria were set up with highly stringent parameters seeking a transcription binding site within $1 \mathrm{~kb}$ upstream of target genes. The binding site of transcription factor AP2 for kasII was identified with a $p$-value of less than $1 \times 10^{-6}$. The conserved sequence of the AP2 binding site was also found in (-) strand GTCTTTCTTTTCTTCTT TTT or CAGAAAGAAAAGAAGAAAAA in (+) strand. Similarly, the putative transcription factor binding site of B3 was identified with a $p$-value cut-off of less than $1 \times 10^{-6}$ on the $1.0 \mathrm{~kb}$ upstream region of $f a d 3$, and the binding site sequence is AAGGAAAAACCA AGAAATA (Fig. 7). This presumably indicated that both kasII and fad3 genes are regulated by AP2/B3 transcription factor families, which are responsible for abiotic stress environments such as drought and 

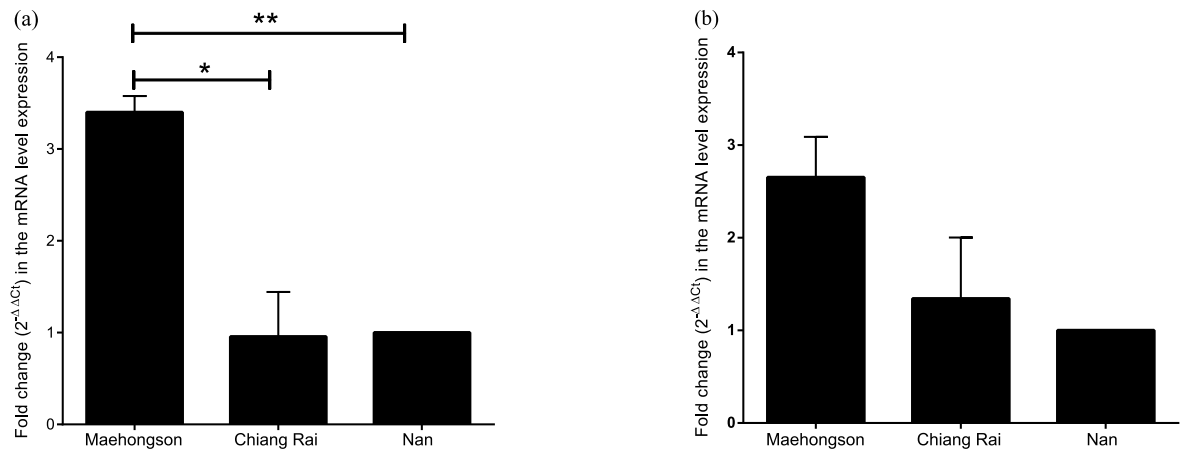

Fig. 1 The mRNA expression of (a) kasII and (b) fad3 in mature perilla seeds collected from different locations. The sample from Nan was set as a control group. The fold change represented the mean \pm SE of duplicate experiments, ${ }^{*} p<0.05, * * p<0.01$.
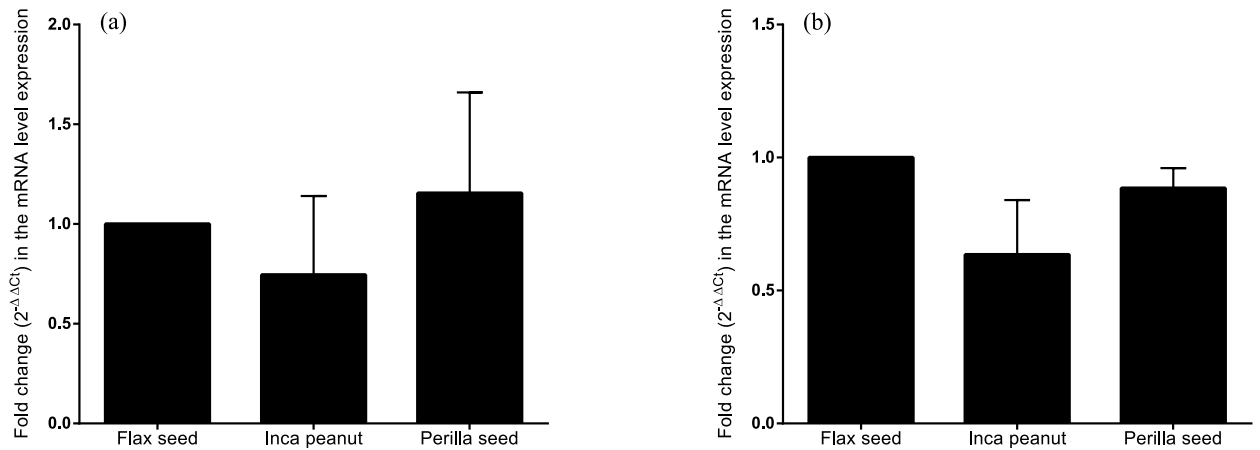

Fig. 2 The mRNA expression of (a) kasII and (b) fad3 in different mature oilseeds. A flax seed sample was set as the control group. The fold change represented the mean \pm SE of duplicate experiments.

low temperature.

\section{DISCUSSION}

Perilla is remarkably known for its high ALA production. In Thailand, the cultivation areas are distributed in the Northern regions, especially in mountain areas. Regarding this, the correlation between ALA productivity and growing locations was determined through the expression of key genes, kasII and fad3, encoding for fatty acid biosynthesis in mature seeds.

In terms of genetic factors, several genes encoding enzymes involving lipid biosynthesis are expressed differently during seed development of oilproducing plants ${ }^{9,19}$. In particular, a number of genes contributing to the production and accumulation of lipids are up-regulated. The transcriptome analysis of inca peanut seeds revealed that kas genes were highly expressed in middle-to-late stages during seed development, where the oil accumulation was beginning ${ }^{10,20}$. In flax seeds, kas genes were highly expressed at the middle stage of embryo development ${ }^{19}$. This expression pattern of kas genes was in agreement with the occurrence in other oil crops such as $J$. curcas $^{21}$. Recent transcriptome analysis of genes contributing lipid biosynthesis in perilla has indicated 43 key genes involved ${ }^{9}$.

To produce ALA at a high level, the desaturase enzymes need to be active. The mutation of fad 2 and fad 3 genes could affect the desaturation process in developing seeds ${ }^{22}$. In particular, the mutation of fad3 gene significantly resulted in the reduction of ALA in flax ${ }^{23}$. In the embryonic mature stage of flax seed, the genes encoding fatty acid desaturase in many isoforms were highly expressed ${ }^{19}$. Particularly, it had been reported that the expressions of the genes encoding sad, fad 2 , and $\mathrm{fad} 3$ were high, which was closely associated with the high production and accumulation of oil in storage cells of inca peanut ${ }^{10}$. These finding were also in good-agreement with the high expression of $f a d 3$ gene in perilla. The increase of ALA to LA ratio during perilla seed development indicated that the $n-3$ desaturase was very active in the middle stage of the developing seed and then sharply declined in the late stage before seed maturation ${ }^{9}$. It was also found that the content of 


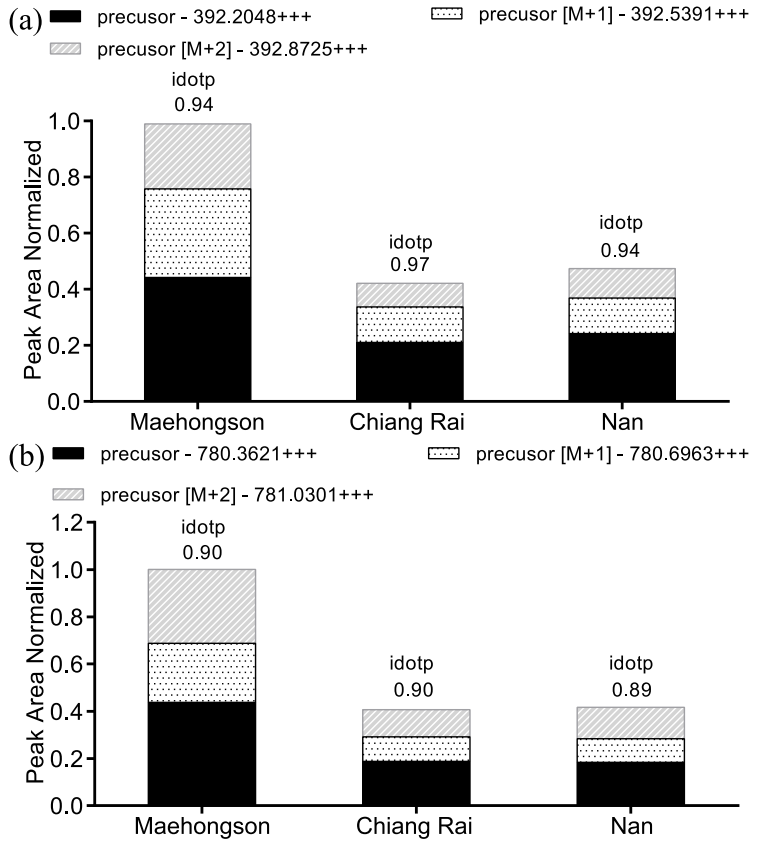

(c)

MAACMSVTCERESGVSALSTTSSNSRRLTKWAAHRRKRVLAKCAPRIRNLVDSSLSLEPRHHLHKSHEC FFGFESRNAPMQRGRQKLLLHPSAYSGEMMAVAVNPAMEVSPKKKPPTKHRRVVVTGMGVETPLGSDPDV FYNNLLEGVSGISEIEAFDCSQFPTRIAGEIKSFSTDGWVVPKLSKRMDKEMLYMLTAGKKALADGGIT VDAMDELNKAKCGVLIGSAMGGMKVFYDAIEALRVSYRKMNPFCVPFATTNMGSAMLAMDLGWMGPNYS ISTACATSNFCI INAANHI IRGEADLMLCGGSDAAI I P IGLGGFVACRALSQRNSDPTKA.SRPWDSNRD GFVMGEGAGVLLLEELEHAKSRGATIYAEFLGGSFTSDAYHMTEPHPQGTGVILCLEKALAQSGVSKED VNY INAHATSTPAGDLKEYQALLHCFGKNPELRVNSTKSMIGHLLGAAGAVEAVATVQAIRTGWVHPNI NLENPDGGVDANVLVGPTKERLDIKVALSNSFGFGGHNSSILFAPYK

Fig. 3 The tryptic peptides represent the protein expression of KASII in mature perilla seeds collected from different locations. Peak areas of tryptic peptides analysed by Skyline, (a) FMLYMLTAGA and (b) GFVMGEGAVLLEELEHAK. The peptide mapping in protein KASII was demonstrated in (c).

ALA reached a steady state and remained constant throughout the late stage of seed development until the end of seed maturation. The evidence of RNA accumulation in mature dry seed was revealed in rice $^{24,25}$. The remaining mRNA transcripts in the mature dry seeds of perilla were possibly due to stored mRNAs required for lipid metabolism such as membrane formation during seed germination. Similarly, we hypothesized that mRNAs could also be accumulated in mature perilla seed at a basal level. According to the results, the expression of kasII and fad3 was found but it was not significantly different among the three areas. However, intensive study of mRNA accumulation in dry perilla seed has not been investigated. Our findings could provide preliminary data on key genes; thus further work

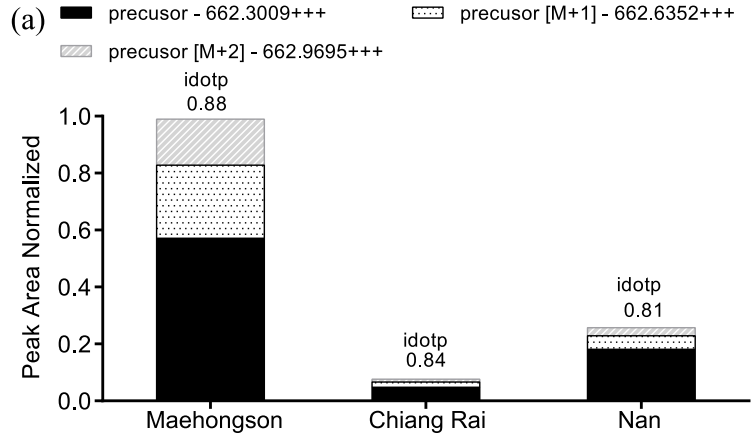

(b)

MAVSSGARLSKSGADGEVFDGQQQYEGIGKRAADKFDPAAPPPFKIADIRAAIPAHCWVK NPWRSLSYVVWDVAAVFALLAAAVY INSWAFWPVYWIAQGTMFWALFVLGHDCGHGSFSD NTTLNNVVGHVLHSS I LVPYHGWRISHRTHHQNHGHVEKDESWVPLPENLYKKLDFSTKF LRYKIPFPMFAYPLYLWYRSPGKTGSHFNPYSDLFKPNERGLIVTSTMCWAAMGVFLLYA STIVGPNMMFKLYGVPYLIFVMWLDTVTYLHHHGYDKKLPWYRSKEWSYLRGGLTTVDQD YGFFNKI HHDIGTHVIHHLFPQI PHYHLVEATREAKRVLGNYYREPRKSGPVPLHLIPAL LKSLGRDHYVSDNGDIVYYQTDDELFPSKKI

Fig. 4 The tryptic peptides represent the protein expression of FAD3 in mature perilla seeds collected from different locations. Peak areas of tryptic peptides analysed by Skyline, SGADGEVFDGQQQYEGIGK (a). The peptide mapping in protein FAD3 was demonstrated in (b).

needs to be carried out on each stage of developing perilla seeds and their mature stage in more cultivation areas.

On the other hand, the altitudes may be one of many factors affecting the productivity of ALA in perilla. As perilla is a short-day plant, it needs a long-night period to induce flowering and cold stress for polyunsaturated fatty acid produc$\operatorname{tion}^{26,27}$. Thus during the growing season of shortday length, the terrain provides a proper low temperature and dry conditions ${ }^{28}$. Usually, the annual average temperatures of Thailand in high mountain areas are low and the lowest yearly temperatures are in December. The exposure of plants to low temperatures during seed maturation and lipid accumulation could then shape the profile of polyunsaturated fatty acids in many developing oil seeds ${ }^{10,29}$. The production of ALA possibly resulted from the interaction of many factors including climate, soil types and conditions, agricultural conditions, genetic diversity, and the ability of plants to adapt to different environments ${ }^{27,29,30}$. In addition, future work should also focus on the question of geographical influences such as altitude, temperature, day-light hours, and humidity on perilla ALA production in more cultivation areas. 


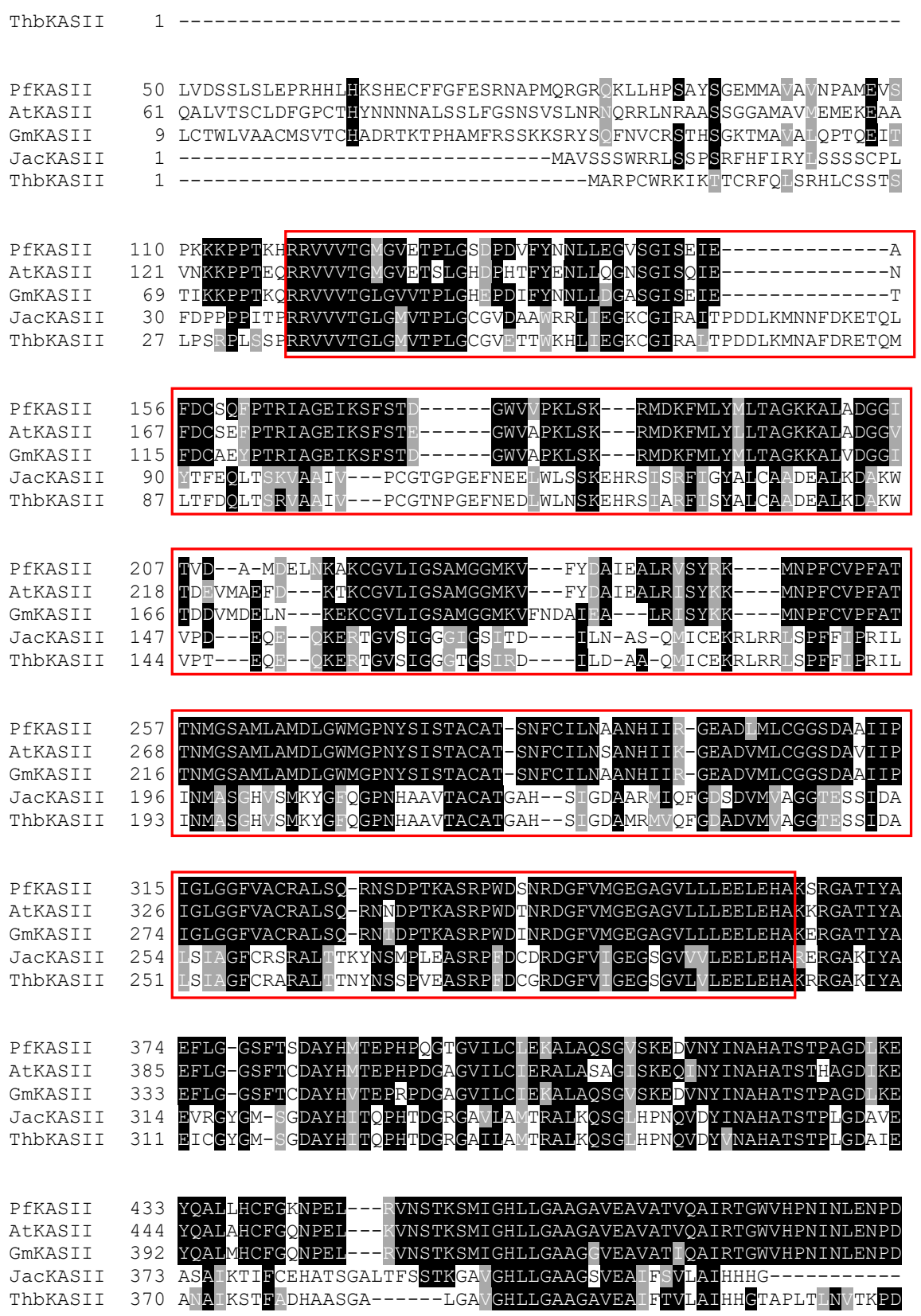

Fig. 5 Multiple alignment of KASII amino acid sequence of $P$. frutescens. KASII protein sequence similarity of P. frutescens (PfKASII) were compared with A. thaliana (AtKASII) and other oilseeds including J. curcas (JacKASII), G. max (GmKASII), and T. cacao (ThbKASII). The results demonstrated that PfKASII has more than $97 \%$ similarity compared with AtKASII. Dark and grey shading indicates high and low consistency amino acid residues, respectively.

Although the expression of the gene encoding fad 3 may reflect the activity of n-3 desaturase and the accumulation of n-3 fatty acids, the production of ALA may also depend on protein abundance. Targeted proteomics were further used to determine protein abundance related to target genes. It was found that the protein abundance of both KASII and FAD3 in Maehongson perilla seeds was higher than that of Chiang Rai and Nan perilla seeds. Presumably, the higher altitudes might play a role in increasing ALA contents due to lower temperature and drought. The increase of KASII and FAD3 expression could be attributed to the increase of drought, which then contributes to increasing n-3 


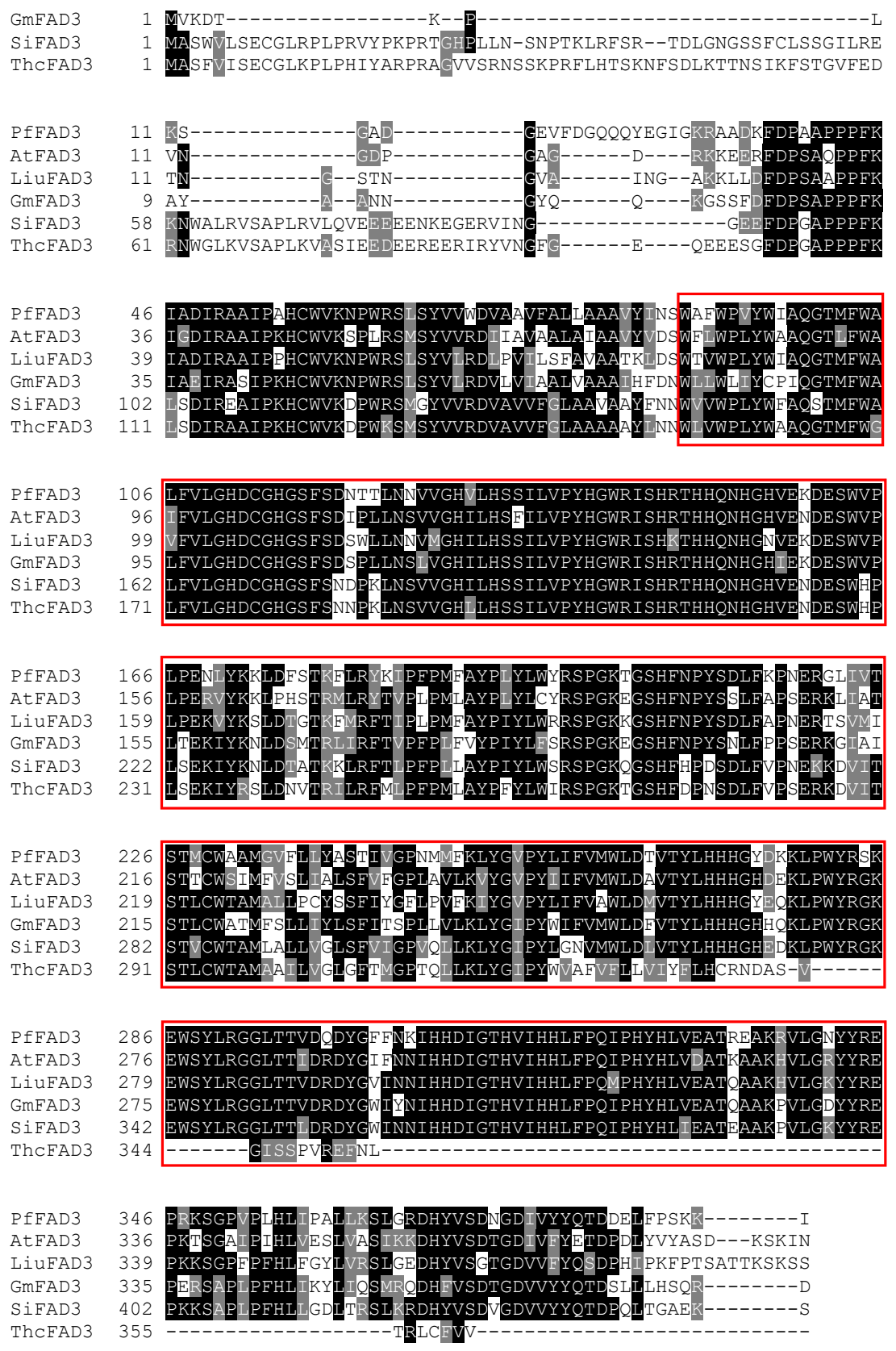

Fig. 6 Multiple alignment of the FAD3 amino acid sequence of $P$. frutescens. FAD3 protein sequence similarity of $P$. frutescens (PfFAD3) were compared with A. thaliana (AtFAD3) and other oil seeds including L. usitatissimum (LiuFAD3), G. max (GmFAD3), S. indicum (SiFAD3), and T. cacao (ThcFAD3). The results demonstrated that PfFAD3 has $95 \%$ similarity compared with AtFAD3. Dark and grey shading indicates high and low consistency amino acid residues, respectively.

fatty acid production. During seed oil accumulation, a number of transcription factors play crucial roles in the regulation of gene expression of lipid synthetic genes. In this regard, the bioinformatics tool was used to predict the transcription factor binding sites which are responsible for the seed oil biosynthesis. The transcription factor binding sites at $1.0 \mathrm{~kb}$ upstream of kasII and fad 3 genes were found to be AP2 and B3 families, respectively. In particular, the AP2 and B3 transcription factors themselves play many roles in seed oil deposition, especially in the seed oil biosynthesis pathway ${ }^{13}$. 
(a) KASII

GTGTGAATTTAATGAATTTGAGGGGTCTATTATATTTATTAGTCAGCTTTIAACGTATCAA ATCCATGACTTTTATCAATGTITITTCITCTTITCTTTCTGGATCTACTTCCCAATACCA TCACCGGACCGGACCACTTGATGATCTTCCTATTTATGAACACTACTACTAGTAAACTCA FAD3

TAACTAAAATTTCAAGAAATATTGTATTTGGTGCATAATACATTGGGGTTGGTAGATTC CCATAGTAAGAAATGAATATAATTTAGTTGGTATACTTGGTACGTGCAAGGAAAAACCAA GAAATATTCATCTTCTTATCACAATTGCTTATATTAGGAAATAAATCAGAGAAATATATT CTTAACTTACACGAATTGAAATGGTGAAACAAATTATGGTAAAGTTTTGTTCTTCTTAAA

(b)

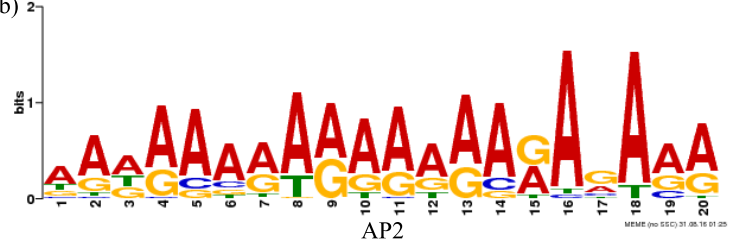

(c)

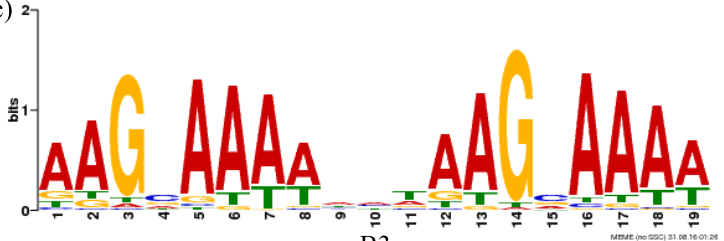

B3

Fig. 7 The DNA binding site motif of AP2/B3 transcription factor within $1.0 \mathrm{~kb}$ upstream of AtKASII and AtFAD3; (a) transcription factor binding site was predicted by transcription factor prediction tool from planttfdb.cbi.pku. edu.cn with a $p$-value less than $1 \times 10^{-6}$, boxed sequences are putative AP2 and B3 binding location and sequence for KASII and FAD3, respectively. (b) AP2 binding site was discovered at position 454-471 bp for kasII gene, and (c) B3 binding site at position 768-786 bp upstream of fad3 gene.

Regarding the abiotic stress environment, a previous study also mentioned that the expression of FAD3 is responsible for the response of $A$. thaliana to drought and subsequently resulted in an increase of ALA production ${ }^{31}$. The explanation of this matter could be relevant to the increase of gene functions in cellular components in order to generate a high level of polyunsaturated fatty acids to incorporate into membrane lipid components which are a tolerance response to drought stress ${ }^{32}$. In fact, the AP2 and B3 families not only have major roles in seed oil biosynthesis but also dominantly respond to drought, high-salt content, and temperature change in oil seeds ${ }^{33}$. As mentioned previously, the possibility that the increase of kasII and fad3 expression corresponding to the increment of ALA production may be associated with the altitude of growing sites and can be explained by the counteraction of perilla to environmental conditions such as drought and low temperature ${ }^{27}$.
In conclusion, the MS-based proteomic analysis confirmed the high expression of KASII and FAD3 in seed samples from Maehongson. It was also found that the transcription factor binding sites AP2 and B3 were specific for kasII and fad3, respectively. These connections might be responsible for a sensitive response to environmental changes in which the increase of kasII and fad3 expression and the ALA production were possibly associated with the altitudes of cultivation areas. This finding would also provide useful information for further genetic manipulations as well as plant treatments in ALA producing-perilla. Furthermore, the increase of ALA productivity in limited areas and restricted conditions could play a part in adding value to the product for further industrial applications.

Acknowledgements: This study was funded by grant number R020058217038 University of Phayao, The Plant Genetic Conservation Project under the Royal Initiation of Her Royal Highness Princess Maha Chakri Sirindhorn (RSPG) and ARDA. This work was also partly supported by Toray foundation year 2017. We are thankful to Ms. Patphorn Chankong for technical support and the Central lab unit for providing instruments in this study.

\section{REFERENCES}

1. Rao S, Abdel-Reheem M, Bhella R, McCracken C, Hildebrand D (2008) Characteristics of high $\alpha$ linolenic acid accumulation in seed oils. Lipids 43, 749-755.

2. Lee JM, Lee H, Kang S, Park WJ (2016) Fatty acid desaturases, polyunsaturated fatty acid regulation, and biotechnological advances. Nutrients 8, 23, 1-13.

3. Ide T, Kobayashi H, Ashakumary L, Rouyer IA, Takahashi Y, Aoyama T, Hashimoto T, Mizugaki M (2000) Comparative effects of perilla and fish oils on the activity and gene expression of fatty acid oxidation enzymes in rat liver. Biochim Biophys Acta 1485, 23-35.

4. Zhao G, Etherton TD, Martin KR, West SG, Gillies PJ, Kris-Etherton PM (2004) Dietary $\alpha$-linolenic acid reduces inflammatory and lipid cardiovascular risk factors in hypercholesterolemic men and women. $J$ Nutr 134, 2991-2997.

5. Simopoulos AP (2016) An increase in the omega6/omega-3 fatty acid ratio increases the risk for obesity. Nutrients 8, 128, 1-17.

6. Innis SM (2008) Dietary omega 3 fatty acids and the developing brain. Brain Res 1237, 35-43.

7. Lukiw WJ, Cui J-G, Marcheselli VL, Bodker M, Botkjaer A, Gotlinger K, Serhan CN, Bazan NG (2005) A role for docosahexaenoic acid-derived neuroprotectin D1 in neural cell survival and Alzheimer disease. $J$ Clin Invest 115, 2774-2783. 
8. Suttajit M, Khanaree C, Tantipaiboonwong P, Pintha K (2015) Omega-3, omega- 6 fatty acids and nutrients of Nga-mon seeds in Northern Thailand. Naresuan Phayao J 8, 80-86.

9. Kim HU, Lee K-R, Shim D, Lee JH, Chen GQ, Hwang $S$ (2016) Transcriptome analysis and identification of genes associated with $\omega-3$ fatty acid biosynthesis in Perilla frutescens (L.) var. frutescens. BMC Genomics 17, 474, 1-18.

10. Wang X, Liu A (2014) Expression of genes controlling unsaturated fatty acids biosynthesis and oil deposition in developing seeds of Sacha Inchi (Plukenetia volubilis L.). Lipids 49, 1019-1031.

11. Hwang S-K, Kim K-H, Hwang Y-S (2000) Molecular cloning and expression analysis of 3-ketoacyl-acp synthases in the immature seeds of Perilla frutescens. Mol Cells 10, 533-539.

12. Chung C-H, Kim J-L, Lee Y-C, Choi Y-L (1999) Cloning and characterization of a seed-specific $\omega-3$ fatty acid desaturase cDNA from Perilla frutescens. Plant Cell Physiol 40, 114-118.

13. Liao B, Hao Y, Lu J, Bai H, Guan L, Zhang T (2018) Transcriptomic analysis of Perilla frutescens seed to insight into the biosynthesis and metabolic of unsaturated fatty acids. BMC Genomics 19, 213, 1-14.

14. Mornkham T, Wangsomnuk P, Fu Y-B, Wangsomnuk P, Jogloy S, Patanothai A (2013) Extractions of high quality RNA from the seeds of Jerusalem artichoke and other plant species with high levels of starch and lipid. Plants 2, 302-316.

15. Untergasser A, Cutcutache I, Koressaar T, Ye J, Faircloth BC, Remm M, Rozen SG (2012) Primer3new capabilities and interfaces. Nucleic Acids Res 40, e115, 1-12.

16. Krishnakumar V, Hanlon MR, Contrino S, Ferlanti ES, Karamycheva S, Kim M, Rosen BD, Cheng C-Y, et al (2015) Araport: the arabidopsis information portal. Nucleic Acids Res 43, 1003-1009.

17. Jin J, He K, Tang X, Li Z, Lv L, Zhao Y, Luo J, Gao $G$ (2015) An arabidopsis transcriptional regulatory map reveals distinct functional and evolutionary features of novel transcription factors. Mol Biol Evol 32, 1767-1773.

18. Jin J, Tian F, Yang D-C, Meng Y-Q, Kong L, Luo J, Gao G (2017) PlantTFDB 4.0: toward a central hub for transcription factors and regulatory interactions in plants. Nucleic Acids Res 45, 1040-1045.

19. Venglat P, Xiang D, Qiu S, Stone SL, Tibiche C, Cram D, Alting-Mees M, Nowak J, et al (2011) Gene expression analysis of flax seed development. BMC Plant Biol 11, 74, 1-14.

20. Wang X, Xu R, Wang R, Liu A (2012) Transcriptome analysis of Sacha Inchi (Plukenetia volubilis L.) seeds at two developmental stages. BMC Genomics 13, 716, $1-16$.

21. Xu R, Wang R, Liu A (2011) Expression profiles of genes involved in fatty acid and triacylglycerol synthesis in developing seeds of Jatropha (Jatropha curcas L.). Biomass Bioenergy 35, 1683-1692.

22. Ohlrogge J, Browse J (1995) Lipid biosynthesis. Plant Cell 7, 957-970.

23. Thambugala D, Duguid S, Loewen E, Rowland G, Booker H, You FM, Cloutier S (2013) Genetic variation of six desaturase genes in flax and their impact on fatty acid composition. Theor Appl Genet 126, 2627-2641.

24. Howell KA, Narsai R, Carroll A, Ivanova A, Lohse M, Usadel B, Millar AH, Whelan J (2009) Mapping metabolic and transcript temporal switches during germination in rice highlights specific transcription factors and the role of RNA instability in the germination process. Plant Physiol 149, 961-980.

25. Sano N, Ono H, Murata K, Yamada T, Hirasawa T, Kanekatsu M (2015) Accumulation of long-lived mRNAs associated with germination in embryos during seed development of rice. J Exp Bot 66, 4035-4046.

26. Khodakovskaya M, McAvoy R, Peters J, Wu H, Li $Y$ (2006) Enhanced cold tolerance in transgenic tobacco expressing a chloroplast $\omega-3$ fatty acid desaturase gene under the control of a cold-inducible promoter. Planta 223, 1090-1100.

27. Xue Y, Chen B, Win AN, Fu C, Lian J, Liu X, Wang R, Zhang X, et al (2018) Omega-3 fatty acid desaturase gene family from two $\omega-3$ sources, Salvia hispanica and Perilla frutescens: cloning, characterization and expression. PLoS One 13, e0191432, 1-25.

28. Lee JK, Ohnishi O (2001) Geographic differentiation of morphological characters among Perilla crops and their weedy types in East Asia. Breeding Sci 51, 247-255.

29. Ding Y, C MN, Hu Y, Shi L, Ma C, Liu Y-J (2012) Characterization of fatty acid composition from five perilla seed oils in China and its relationship to annual growth temperature. J Med Plants Res 6, 1645-1651.

30. Siriamornpun S, Li D, Yang L, Suttajit S, Suttajit M (2006) Variation of lipid and fatty acid compositions in Thai Perilla seeds grown at different locations. Songklanakarin J Sci Technol 28, 17-21.

31. Yuan S-w, Wu X-l, Liu Z-h, Luo H-b, Huang R-z (2012) Abiotic stresses and phytohormones regulate expression of fad 2 gene in Arabidopsis thaliana. $J$ Integr Agric 11, 62-72.

32. Gigon A, Matos A-R, Laffray D, Zuily-Fodil Y, PhamThi A-T (2004) Effect of drought stress on lipid metabolism in the leaves of Abidopsis thaliana (Ecotype Columbia). Ann Bot 94, 345-351.

33. Dossa K, Wei X, Li D, Fonceka D, Zhang Y, Wang $\mathrm{L}$, Yu J, Boshou L, et al (2016) Insight into the AP2/ERF transcription factor superfamily in sesame and expression profiling of DREB subfamily under drought stress. BMC Plant Biol 16, 171, 1-13. 